\title{
On the Comparison of Predictive Control and Command Governor approaches for operational management of drinking water networks: A case study ${ }^{\star}$
}

\author{
Francesco Tedesco* Carlos Ocampo-Martinez ${ }^{* *}$ \\ Alessandro Cassavola* Vicenç Puig ${ }^{* *}$ \\ * University of Calabria, DIMES, \\ 87036 Rende (CS), Italy, \\ \{casavola, ftedesco\}@dimes.unical.it \\ ** Automatic Control Department, Universitat Politècnica de \\ Catalunya (UPC), Institut de Robòtica i Informàtica Industrial \\ (CSIC-UPC), Llorens i Artigas 4-6, 08028 Barcelona, Spain \\ \{cocampo, vpuig\}@iri.upc.edu
}

\begin{abstract}
This paper evaluates the application of command governor (CG) strategy for the operational control of drinking water networks (DWN) given their large-scale and complex nature, the permanent and relevant effect of the disturbances (water demands) and their marginal stability feature. Moreover, the performance improvement offered by CG is compared with the application of model predictive control for the same management purposes and in the same context. The paper also discusses the effectiveness of both strategies and highlights the advantages of each approach. The Barcelona DWN is considered as case study for the undertaken assessment analysis.
\end{abstract}

\section{INTRODUCTION}

Drinking Water Networks (DWNs) are in charge of bringing water from production plants to consumers. These networks are of paramount importance for the convenient functioning of modern society. Hence guaranteeing the service in a sustainable manner is a must in these networks. From a dynamical systems point of view, DWNs are large-scale multi-input/multi-output flow systems that must be reliable and resilient to operational constraints and continuously varying conditions of both deterministic and probabilistic nature. Customers behaviour influences the transport and storage operations within the network. Water use can vary in both the long and the short term, usually presenting time-based patterns for different areas. Therefore, a better understanding and forecasting of water demands will improve modelling and control of DWNs.

The growing complexity of these network systems, i.e., dimensionality, information structure constraints, nonlinearities, uncertainty, and the demand for higher performance make their management costly to be solved in real-time and the corresponding control problem has become an increasingly hot environmental and socioeconomic research subject worldwide. Different approaches reported in the literature highlight the relevance of the topic. As discussed in Pulido-Calvo and GutiérrezEstrada [2011], during the last years optimal operation of water supply systems has been addressed by a

\footnotetext{
* This work has been partially supported by the EU Project EFFINET (FP7-ICT-2011-8-31855) and the DGR of Generalitat de Catalunya (SAC group Ref. 2009/SGR/1491).
}

wide variety of methods, ranging from heuristics and expert systems to more advanced mathematical modelling and optimization techniques such as linear programming, dynamic programming, non-linear programming, hierarchical-decompositions, combinatorial schemes, and more recently, model predictive control (MPC). Examples of some of the aforesaid methods are reported in Castelletti et al. [2012], Cembrano et al. [2011], El Mouatasim et al. [2012], Ocampo-Martinez et al. [2013b], Pulido-Calvo et al. [2012], Vieira et al. [2011], Grosso et al. [2013], among many others.

Among the aforementioned approaches, decision policies inspired by the MPC framework [Maciejowski, 2002], are suitable to face the operation of DWNs due to their flexibility to manage constraints and minimize the multiobjective cost indexes found in the optimization of these complex systems, see Ocampo-Martinez et al. [2013a]. The basic idea of MPC is to exploit a model of the system to predict its future evolution and compute control actions by optimising a desired cost function that takes into account such predictions. If future disturbances can be estimated and their uncertainty modeled, a robust MPC can be developed to explicitly consider their effect on the future evolution of the controlled system.

A further family of constrained control strategies based on the same receding horizon paradigm worth mentioning is that of Command Governors (CG). The CG approach can be regarded as a constrained supervision strategy that computes its actions by solving on-line a constrained convex optimization problem based on future system predictions, similarly to any MPC strategy, but with the remarkable 
difference that the stability of the closed-loop system is not a concern. In fact, a CG unit is a nonlinear static module added to an asymptotically stable plant in charge of modifying, whenever necessary, the prescribed command signal when its unmodified application would lead to constraint violations and, in turn, possibly loss of stability. This modification is typically achieved by solving on-line at each time instant a constrained optimization problem, whose constraints take into account future system predictions. As in MPC schemes, a modified command sequence is computed at each sampling instant and, according to the receding horizon control ( $\mathrm{RHC}$ ) policy, only the first sample of such a sequence is applied and the entire procedure is repeated at the next time instant.

CG usage can be justified in typical industrial applications wherein a massive amount of flops per sampling time is not allowed, and/or one is only commissioned to add to existing standard PID-like compensators peripheral units which, as CGs, do not change the primal compensated control system. Studies along these lines can be found in Casavola and Mosca [1996], Bemporad et al. [1997]. For specific results on CG applied to nonlinear systems see Angeli and Mosca [1999]. Moreover, for CGs approaches from different perspectives, see Kapasouris et al. [1990], Gilbert et al. [1995].

At the best of the authors knowledge, the CG approach has never been applied for the management and control of DWNs. Therefore, the main contribution of this paper is the assessment of the application of both (MPC and CG) strategies when considering the operational management of a large-scale complex system represented by a DWN. From this assessment, several interesting aspects arise such as the level of performance reachable by taking into account the management criteria and operative constraints of the network and the suitability of standard CG formulations to deal with marginally stable systems.

The organization of the paper is as follows. The problem of the operational control of DWNs is stated in Section 2. The MPC and CG approaches to address such a problem are recalled in Section 3. Comparison results achieved by using the Barcelona DWN as case study are presented in Section 4. Finally, some conclusions and future research lines are presented in Section 5.

\section{OPERATIONAL CONTROL OF DWN}

\subsection{Control-oriented Modelling}

Here the DWN modeling approach proposed in OcampoMartinez et al. [2013b] is briefly recalled. A general DWN can be represented by a directed graph $G(\mathcal{V}, \mathcal{E})$, where a set of elements, i.e., $n_{s}$ sources, $n_{x}$ storage elements, $n_{q}$ intersection nodes and $n_{d}$ sinks represent the vertices $v \in \mathcal{V}$ that are connected by direct links $a \in \mathcal{E}$. For the network operation, water is transported along the links by $n_{u}$ flow actuators (i.e., pipes and valves), passing through reservoirs or tanks, from specific origin locations to specific destination locations. The network is subject to several capacity and operational constraints, and to measured stochastic flows to sinks driven by customers water demand.
Collecting the volumes of water contained in the storage elements in the state vector $\mathbf{x}_{k} \in \mathbb{R}^{n_{x}}$, the flows through the actuators in the manipulated input vector $\mathbf{u}_{k} \in \mathbb{R}^{n_{u}}$, and considering the demanded flows additive measurable disturbances $\mathbf{d}_{k} \in \mathbb{R}^{n_{d}}$, the control-oriented model of the DWN $G(\mathcal{V}, \mathcal{E})$ may be abstracted and described by the following set of linear (or linearised) discrete differencealgebraic equations (DAE) for all time instant $k \in \mathbb{N}$ :

$$
\begin{aligned}
\mathbf{x}_{k+1} & =\mathbf{A x}_{k}+\mathbf{B} \mathbf{u}_{k}+\mathbf{B}_{d} \mathbf{d}_{k}, \\
\mathbf{0} & =\mathbf{E}_{u} \mathbf{u}_{k}+\mathbf{E}_{d} \mathbf{d}_{k},
\end{aligned}
$$

where the difference equations in (1a) describe the dynamics of storage tanks, and the algebraic equations in (1b) describe the static relations (i.e., mass balance at junction nodes) in the network. Moreover, $\mathbf{A}, \mathbf{B}, \mathbf{B}_{d}, \mathbf{E}_{u}, \mathbf{E}_{d}$, are time-invariant matrices of suitable dimensions dictated by the network topology.

The system is subject to hard state and input constraints given by convex polytopic sets defined as

$$
\begin{aligned}
& \mathcal{X} \triangleq\left\{\mathbf{x}_{k} \in \mathbb{R}^{n_{x}} \mid \mathbf{G x}_{k} \leq \mathbf{g}\right\} \subset \mathbb{R}^{n_{x}} \quad \forall k, \\
& \mathcal{U} \triangleq\left\{\mathbf{u}_{k} \in \mathbb{R}^{n_{u}} \mid \mathbf{F} \mathbf{u}_{k} \leq \mathbf{f}\right\} \subset \mathbb{R}^{n_{u}} \quad \forall k,
\end{aligned}
$$

where $\mathbf{G} \in \mathbb{R}^{c_{x} \times n_{x}}, \mathbf{g} \in \mathbb{R}^{c_{x}}, \mathbf{F} \in \mathbb{R}^{c_{u} \times n_{u}}$, $\mathbf{f} \in \mathbb{R}^{c_{u}}$, being $c_{x}$ and $c_{u}$ the number of state and input constraints, respectively. Moreover, for service reliability, the states are also subject to the following safety constraint:

$$
\mathbf{x}_{k} \geq \mathbf{d}_{\text {net }, k} \quad \forall k,
$$

where $\mathbf{d}_{\text {net, } k} \in \mathbb{R}^{n_{x}}$ is the vector of (possibly timevarying) lower-bounds on water storages (expressed in $\mathrm{m}^{3}$ ) necessary to avoid stock-outs.

\subsection{System Operational Goals}

Different criteria may be taken into account when managing a DWN. In this paper and according to a given context, the operational goals in the management of the DWN are of three kinds: economic, safety, and smoothness, and are respectively stated as follows:

(1) To provide a reliable water supply minimising water production and water transport costs.

(2) To guarantee the availability of enough water in each storage tank to satisfy its underlying stochastic demand.

(3) To operate the DWN under smooth control actions.

The economic and smoothness goals can be achieved by minimising the following performance indicators ${ }^{1}$ :

$$
\begin{aligned}
J_{E, k} & \triangleq\left\|\left(\boldsymbol{\alpha}_{1}+\boldsymbol{\alpha}_{2, k}\right)^{\mathrm{T}} \mathbf{u}_{k}\right\|_{1, \mathbf{w}_{e}}, \\
J_{\Delta U, k} & \triangleq\left\|\Delta \mathbf{u}_{k}\right\|_{2, \mathbf{w}_{u}}^{2},
\end{aligned}
$$

where $J_{E} \in \mathbb{R}_{\geq 0}$ represents the economic cost of network operation taking into account water production cost $\boldsymbol{\alpha}_{1} \in$ $\mathbb{R}^{n_{u}}$ and water pumping cost $\boldsymbol{\alpha}_{2} \in \mathbb{R}^{n_{u}}$, which change every time instant $k$ according to the variable electric tariff; $J_{\Delta U} \in \mathbb{R}_{\geq 0}$ represents the penalisation of control signal variations $\Delta \mathbf{u}_{k} \triangleq \mathbf{u}_{k}-\mathbf{u}_{k-1}$, of use to extend actuators life and assure a smooth operation; $\|\cdot\|_{p, \mathbf{W}}$ is a

\footnotetext{
1 The performance indicators considered in this work may either vary or be generalised with the corresponding manipulation to include other control objectives.
} 
weighted $p$-norm; and $\mathbf{W}_{e}, \mathbf{W}_{u}$ are diagonal matrices that weight each decision variable in the corresponding control objective.

The second goal is enforced by the safety constraint (3), which can be conveniently reformulate as a soft constraint in the following way:

$$
\mathbf{x}_{k} \geq \mathbf{x}_{s, k}-\boldsymbol{\xi}_{k} \geq \mathbf{0} \quad \forall k,
$$

where $\mathbf{x}_{\mathrm{s}} \in \mathbb{R}^{n_{x}}$ is a vector of safety volume thresholds in $\mathrm{m}^{3}$, estimated empirically, above which is desired to keep the reservoirs to cope with the risk involved in the certainty-equivalence principle due to demand uncertainty. Moreover, $\boldsymbol{\xi} \in \mathbb{R}^{n_{x}}$ represents the amount of volume in $\mathrm{m}^{3}$ that goes down from the desired safety thresholds. As a result, the new performance index

$$
J_{S, k} \triangleq\left\|\boldsymbol{\xi}_{k}\right\|_{2, \mathbf{w}_{x}}^{2}
$$

is stated. A proper selection of the safety volumes in $\mathbf{x}_{\mathrm{s}}$ should be done because they effect the conservativeness and the sub-optimality of the solution.

\section{MPC AND CG STRATEGIES APPLIED TO DWN OPERATIONAL CONTROL}

\subsection{MPC for DWN Operational Control}

The main goal of the operational control of DWN at transport level is to satisfy the demands at consumer sides, and optimizing, at the same time, the management policies expressed as a multi-objective optimal control problem. Hence, MPC results a suitable technique to control a DWN because its capability to deal efficiently with multi-variable dynamic constrained systems and predict the proper actions to achieve the optimal performance according to a user-defined cost function. Moreover, the MPC design follows a systematic procedure [Maciejowski, 2002], which generates the control input signals to the plant by combining a prediction model and a receding-horizon control (RHC) strategy.

Problem 1. (MPC for DWNs). Consider the system (1) at a measured condition. Given a prediction horizon $H_{p} \in \mathbb{N}$, and the control objectives (see (4) and (6)) aggregated in a performance index $J: \mathbb{R}^{n H_{p} \times m\left(H_{p}-1\right)} \rightarrow \mathbb{R}$, the MPC problem for DWNs consists in solving a finite horizon optimal control problem (FHOCP) given by

$$
J^{*} \triangleq \min _{\mathbf{u}^{*}, \varepsilon^{*}} \sum_{i=k}^{k+H_{p}-1}\left[J_{E, k}+J_{S, k}+J_{\Delta U, k}\right]
$$

subject to:

$$
\begin{aligned}
& \mathbf{x}(i+1 \mid k)=\mathbf{A x}(i \mid k)+\mathbf{B u}(i \mid k)+\mathbf{B}_{p} \mathbf{d}(i \mid k), \\
& \mathbf{E}_{1} \mathbf{u}(i \mid k)+\mathbf{E}_{2} \mathbf{d}(i \mid k)=\mathbf{0}, \\
& \mathbf{x}_{\min } \leq \mathbf{x}(i+1 \mid k) \leq \mathbf{x}_{\max } \\
& \mathbf{u}_{\min } \leq \mathbf{u}(i \mid k) \leq \mathbf{u}_{\max } \\
& \mathbf{x}(i+1 \mid k) \geq \mathbf{x}_{s}(k)-\boldsymbol{\xi}(i+1 \mid k) \geq \mathbf{0}, \\
& (\mathbf{x}(k \mid k), \mathbf{d}(k \mid k))=(\mathbf{x}(k), \mathbf{d}(k)) .
\end{aligned}
$$

Then, according to the RHC strategy, apply only the first column vector $\mathbf{u}^{*}(k \mid k)$ of the optimal sequence

$$
\underline{\mathbf{u}}_{k}^{*}\left(\mathbf{x}_{k}\right) \triangleq\left[\mathbf{u}^{*}(k \mid k), \ldots, \mathbf{u}^{*}\left(k+H_{p}-1 \mid k\right)\right] .
$$

At the next time instant, the prediction horizon is shifted one time instant ahead and the optimisation is restarted with new feedback measurements and updated predictions to compensate unmeasured disturbances and model inaccuracies. This scheme is repeated at each future time instant.

Remark 1. Despite the intuitive formulation of the RHC strategy, on-line tuning of an MPC controller is not trivial or systematic. The MPC tuning parameters for the given cost function usually are prediction horizon $H_{p}$, control horizon $H_{u}$ and weighting matrices $\mathbf{W}_{e}, \mathbf{W}_{x}, \mathbf{W}_{u}$. These matrices are in general defined as $\mathbf{W}_{i} \triangleq \gamma_{i} \mathbf{I}$, where $\gamma \in \mathbb{R}$ and $\mathbf{I}$ corresponds to an identity matrix of suitable dimensions.

\section{2 $C G$ for $D W N$ operational control}

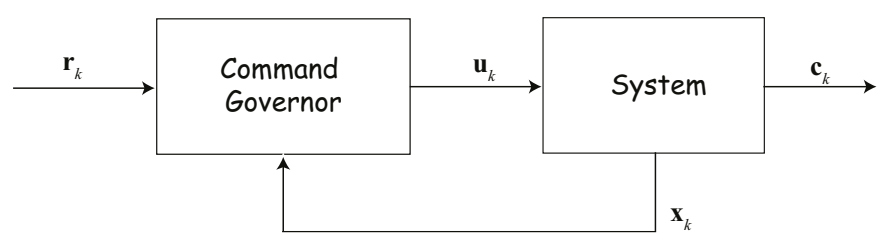

Figure 2. CG Scheme

The CG scheme of interest here is depicted in Figure 2. There, a stable plant is supervised by the CG device via the command sequence $\mathbf{u}_{k}$. In particular, at each time $k$, on the basis of the desired reference $\mathbf{r}_{k}$ and measured state $\mathbf{x}_{k}$, the command $\mathbf{u}_{k}$ is computed as the best approximation of $\mathbf{r}_{k}$ under the following pointwise-in-time constraints:

$$
\mathbf{c}_{k} \in \mathcal{C}
$$

to be held true along the system trajectories generated by the CG, where

$$
\mathbf{c}_{k}:=\mathbf{C x}_{k}+\mathbf{L} \mathbf{u}_{k}
$$

$\mathcal{C}$ is a convex set and $\mathbf{C}:=\left[\mathbf{I}_{n_{x}}^{T}, \mathbf{0}_{n_{u}}^{T}\right]^{T}$ and $\mathbf{L}:=$ $\left[\mathbf{0}_{n_{x}}^{T}, \mathbf{I}_{n_{u}}^{T},\right]^{T}$. For the problem at hand, the set $\mathcal{C}$ is defined

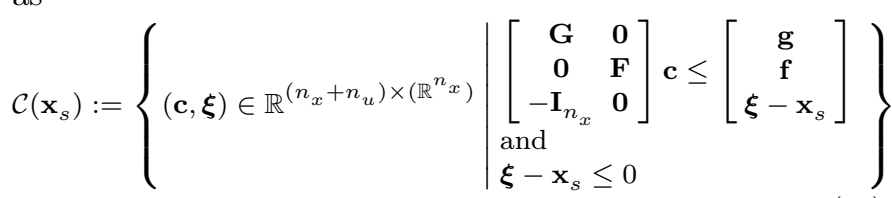

where matrices $\mathbf{G}, \mathbf{F}$ and vectors $\mathbf{g}, \mathbf{f}$ are the same as in (2). The main idea behind the CG approach is that of selecting and applying at each time step a virtual command $\mathbf{u}(i \mid k) \equiv \mathbf{w}, \forall i$, chosen in such a way that, if constantly applied to the system over a semi-infinite horizon $i \in[0, \infty)$, from the initial state $x_{k}$, it never would produce constraint violations. Moreover, in order to take into account the algebraic-equations (1b), the following set is introduced:

$$
\mathcal{W}(\mathbf{d})=\left\{\mathbf{w} \in \mathbb{R}^{n_{u}}: \mathbf{E}_{1} \mathbf{w}+\mathbf{E}_{2} \mathbf{d}=0\right\} .
$$

The applied command $\mathbf{w}$ is chosen in such a way that the future predictions (virtual evolutions) of the c-variable along the virtual time $i$ under a constant virtual command $\mathbf{u}(i \mid k) \equiv \mathbf{w}$ from the initial state $\mathbf{x}$ (at virtual time $i=0$ ) $\mathbf{c}(i, \mathbf{x}, \mathbf{w}, \mathbf{d})=\mathbf{C}\left(\mathbf{A}^{i} \mathbf{x}+\sum_{j=0}^{i-1} \mathbf{A}^{i-j-1}\left(\mathbf{B w}+\mathbf{B}_{d} \mathbf{d}\right)\right)+\mathbf{L w}$ 


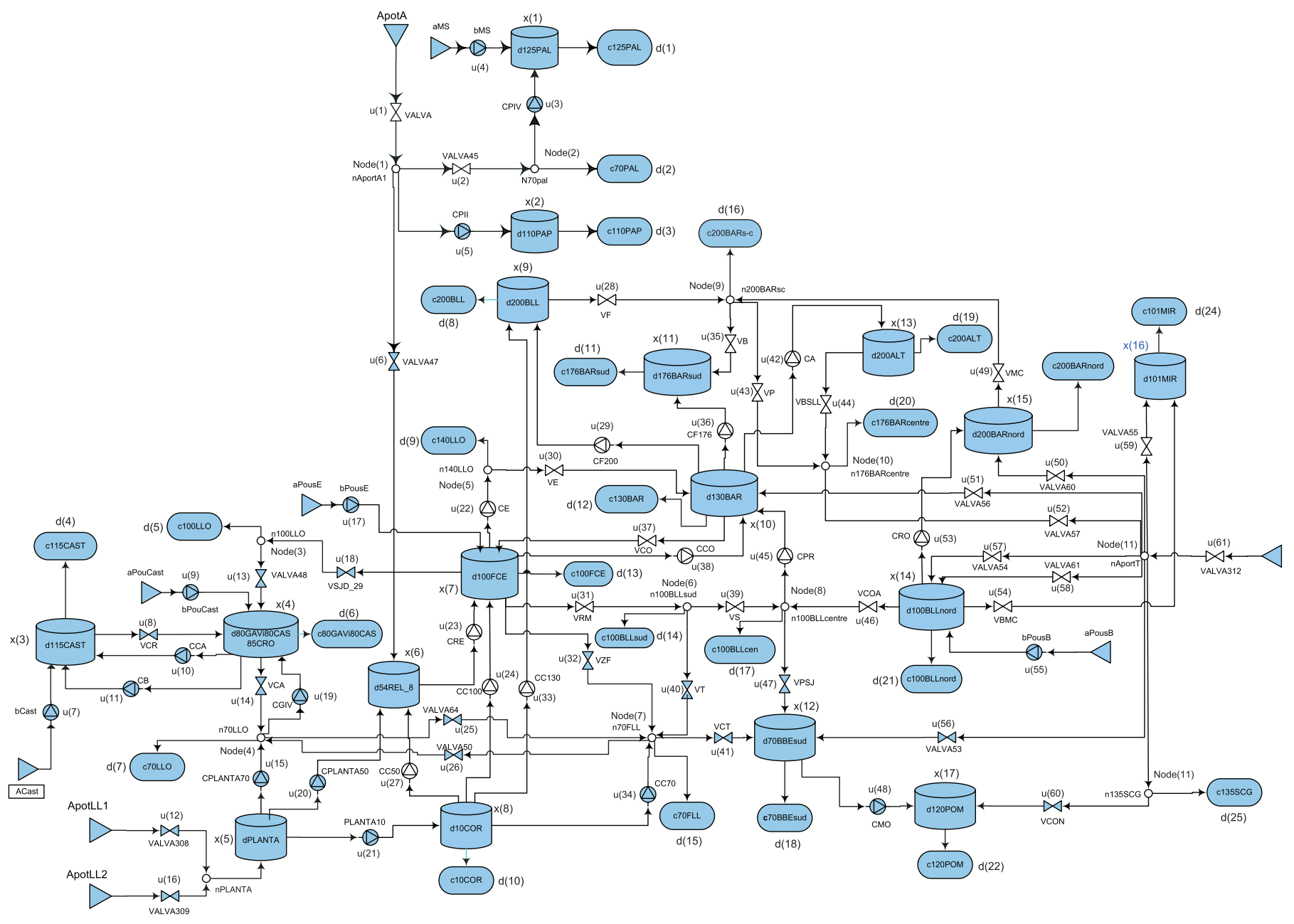

Figure 1. Case Study: Aggregate model of the Barcelona DWN

do not violate constraints $\mathbf{c}(i, \mathbf{x}, \mathbf{w}, \mathbf{d}) \in \mathcal{C}, \forall i \in \mathbb{Z}_{+}$.

In the case of a constant disturbance $\mathbf{d}(i \mid k)=\mathbf{d}$, this is obtained by selecting the applied command as follows:

$\mathbf{w} \in \mathcal{V}(\mathbf{x}, \mathbf{d}):=\{\mathbf{w} \in \mathcal{W}(\mathbf{d}):$

$\exists \boldsymbol{\xi}_{i}$ such that $\left.\left(\mathbf{c}(l, \mathbf{x}, \mathbf{w}, \mathbf{d}), \boldsymbol{\xi}_{i}\right) \in \mathcal{C}\left(\mathbf{x}_{s}\right), \forall i \in \mathbb{Z}_{+}\right\}$.

It is worth mentioning that, if $\mathbf{A}$ is $\operatorname{Schur}$, the $\operatorname{set} \mathcal{V}(\mathbf{x}, \mathbf{d})$, $\forall \mathbf{x} \in \mathbb{R}^{n_{x}}$, is convex and finitely determined, viz. there exists an a priori known integer $i_{0}$ (see Gilbert et al. [1995]) such that if $\mathbf{c}(i, \mathbf{x}, \mathbf{w}, \mathbf{d}) \in \mathcal{C}\left(\mathbf{x}_{s}\right), i \in\left\{0,1, \ldots i_{0}\right\}$, then $\mathbf{c}(i, \mathbf{x}, \mathbf{w}, \mathbf{d}) \in \mathcal{C}\left(\mathbf{x}_{s}\right), \forall i \in \mathbb{Z}_{+}$.

Finally, the CG problem is solved by choosing at each time instant $k$ a command $\mathbf{u}_{k}^{*}$, which is the solution of the following convex optimization problem:

$$
\mathbf{u}_{k}^{*}=\arg \min _{\mathbf{w} \in \mathcal{V}\left(\mathbf{x}_{k}, \mathbf{d}\right)} J^{*},
$$

where $J^{*}$ is computed as in (7a) with

$$
J_{E, k} \triangleq\left\|\left(\boldsymbol{\alpha}_{1}+\boldsymbol{\alpha}_{2, k}\right)^{\mathrm{T}}\left(\mathbf{w}-\mathbf{r}_{k}\right)\right\|_{1, \mathbf{w}_{e}}
$$

In the case of time-varying disturbance predictions, commands $\mathbf{u}(i \mid k)$ need to be selected in a set that depends on the entire disturbance sequence $\underline{\mathbf{d}}_{k} \triangleq\left[\mathbf{d}(0 \mid k), \ldots, \mathbf{d}\left(i_{0} \mid k\right)\right]$ and has the following form:

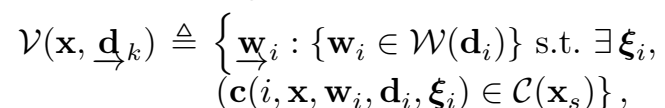

$\forall i \in\left\{0,1, \ldots, i_{0}\right\}$.

The above set can be used also when, as is the case for DWNs, A has simple roots on the unit circle. In this situation, it is possible to find a choice of coordinates which changes $\mathbf{A}$ into the form

$$
\mathbf{A}=\left[\begin{array}{cc}
\mathbf{I}_{d} & \mathbf{0} \\
\mathbf{0} & \mathbf{A}_{s}
\end{array}\right]
$$

where $d$ is the number of characteristic roots $\left|\lambda_{i}\right|=1$ and $\mathbf{A}_{s} \in \mathbb{R}^{\left(n_{x}-d\right) \times\left(n_{x}-d\right)}$ is asymptotically stable. Matrix $\mathbf{C}$ does not change because it is composed by an identity matrix and a zeros matrix.

Then, a problem similar to (13) can be solved in this case and the obtained solution is the sequence

$$
\underset{\mathbf{u}_{k}^{*}}{\rightarrow}=\arg \min _{\mathbf{w} \in \mathcal{V}\left(\mathbf{x}, \underset{\mathbf{d}_{k}}{\rightarrow}\right.} J^{*},
$$

where only the first component will be applied according to the RHC philosophy.

\section{APPLICATION TO THE BARCELONA DWN}

\subsection{Case Study Description}

The selected case study is, without loss of generality, an aggregate and representative version of the entire 
Barcelona DWN. In this aggregate model, some consumer demand sectors of the network are concentrated in a single point. Similarly, some tanks are aggregated in a single element and the respective actuators are considered as a single pumping station or valve [Ocampo-Martinez et al., 2009]. The model consists of 17 tanks, 61 actuators, 25 measured demands and 11 nodes (see Figure 1). Water is taken from rivers Besòs and Ter, particularly from three sources named Abrera, Llobregat and Cardedeu.

\subsection{Closed-loop Setup}

All results have been obtained by considering a four-day real-demand scenarios (with 1 hour of sampling time), and $H_{p}=H_{u}=i_{0}=24$. In the case of both MPC and CG strategies, control objectives in (7a) are prioritised with $\gamma_{e}=100, \gamma_{x}=10$ and $\gamma_{u}=1$, following a trial-and-error tuning strategy. The network has been simulated by using the same model used to design the MPC controller but fed with real water demands. The network model has been calibrated and validated by using real data provided by AGBAR $^{2}$. All simulations have been undertaken by using the Yalmip interpreter [Löfberg, 2004] and the CPLEX solver, all under MATLAB ${ }^{\complement} 8.2$ environment, running on an Intel ${ }^{\circledR}$ Core i5-3330 machine with $3.3 \mathrm{GHz}$ and $8 \mathrm{~GB}$ RAM.

\subsection{Results, Comparison and Discussion}

Both strategies yield quite similar results from both qualitative and quantitative points of view. Minor differences have been observed, mainly related to the statement of the optimization problems for each strategy. In particular, CG achieves a slightly better performance than the MPC strategy in terms of electric cost that nevertheless weakly outperforms CG in terms of water cost (see Table 1). Regarding the total cost, MPC yields to lower values mainly towards the final of the simulation. Finally, the computational burden is practically the same with a slight advantage for the CG setup. Notice that, despite all simulations have considered a four-day scenario, the closed-loop system reached its steady state at the end of the second day of simulations.

Regarding the dynamical evolution of network elements, notice that both approaches fulfill the objective of pumping water during the time periods of cheaper electricity cost (nights), see Figure 4. In some cases, CG manages better the tanks by storing less water than MPC (see Figure 3). However, this fact has no significant consequences in the network operation, as shown in Table 1. The water taken from sources is almost the same for both approaches (except for the transient in Llobregat source, see Figure 5), which in fact leads to similar performance for the considered approaches.

Finally, notice that the economic costs in Table 1 are given in economic units rather than real values (Euro) due to confidentiality reasons.

\footnotetext{
2 Aigües de Barcelona, S.A., the company that manages the Barcelona DWN.
}

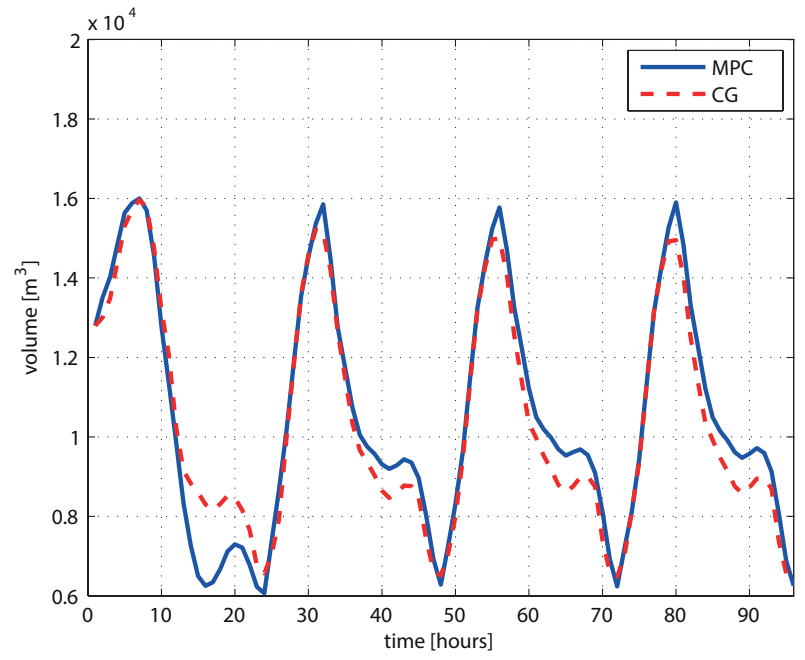

Figure 3. Resultant volume related to tank $x(10)$ (d130BAR)

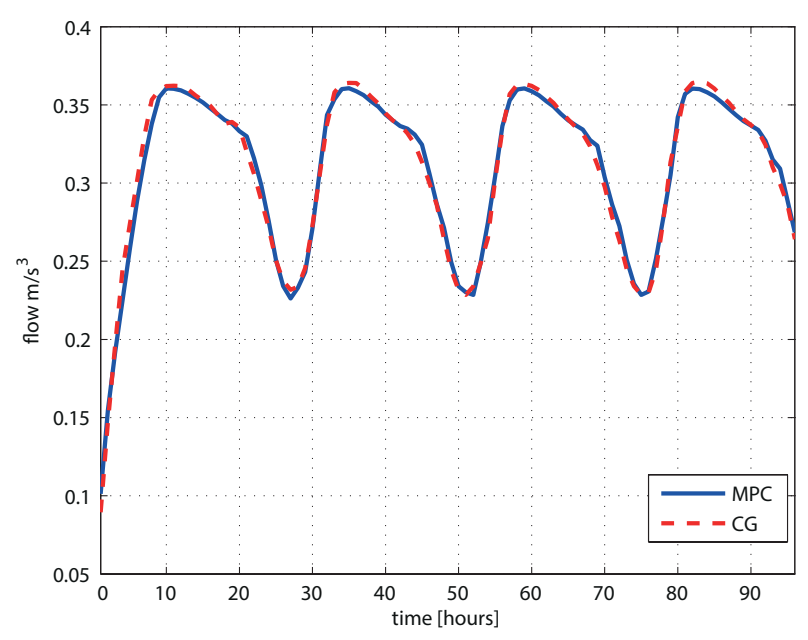

Figure 4. Computed flow related to valve $u(54)$.

\section{CONCLUSIONS}

This paper has presented a comparison between the CG and MPC approaches when applied to the operational control of the aggregate model of the DWN of Barcelona, Spain. The comparative assessment required a slightly modified and expanded CG theory for facing with marginal stable systems, incorporating equality constraints and dealing with disturbance rejection goals. The provided simulative results have shown the similarity of both techniques in managing the system. In particular, both approaches shown similar computational burdens although the CG approach seems to be less demanding (see Table 1). Future works will be devoted to address and solve the problem with distributed CG approaches.

\section{REFERENCES}

D. Angeli and E. Mosca. Command governor for nonlinear systems under constraints. IEEE Trans. on Automatic Control, 44:816-820, 1999.

A. Bemporad, A. Casavola, and E. Mosca. Nonlinear control of constrained linear systems via predictive reference management. IEEE Trans. on Automatic Control, 42(3):340-349, 1997. 
Table 1. Complete break-down of economic costs

\begin{tabular}{ccccccccc}
\hline \multirow{2}{*}{ Day } & \multicolumn{2}{c}{ Water Cost } & \multicolumn{2}{c}{ Electric Cost } & \multicolumn{2}{c}{ Total Cost } & \multicolumn{2}{c}{ CPU Time [s] } \\
& MPC & CG & MPC & CG & MPC & CG & MPC & CG \\
\hline 1 & 14.4652 & 14.4906 & 21.5238 & 21.4424 & 35.9890 & 35.9330 & 9.48 & 9.34 \\
2 & 25.8734 & 26.0831 & 21.6784 & 21.5962 & 47.5519 & 47.6793 & 10.31 & 10.11 \\
3 & 27.5519 & 27.8655 & 21.8691 & 21.7867 & 49.4210 & 49.6522 & 10.71 & 10.23 \\
4 & 27.6296 & 27.9428 & 21.8664 & 21.7846 & 49.4960 & 49.7273 & 10.65 & 10.29 \\
\hline
\end{tabular}
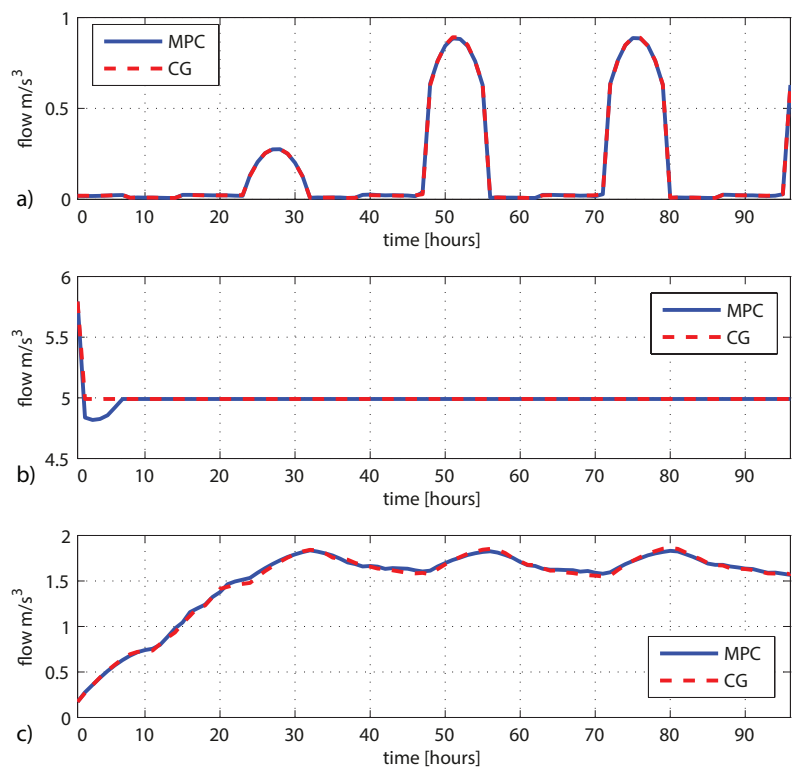

Figure 5. Comparison of the network inflow from the main water sources: a) Abrera $(u(1))$, b) Llobregat $(u(12)+$ $u(16))$, and c) Cardedeu $(u(61))$.

A. Casavola and E. Mosca. Reference governor for constrained uncertain linear systems subject to bounded input disturbances. In Proc. of 35th IEEE CDC, 1996.

A. Castelletti, F. Pianosi, and R. Soncini-Sessa. Stochastic and robust control of water resource systems: Concepts, methods and applications. In System Identification, Environmental Modelling, and Control System Design, pages 383-401. Springer London, 2012.

G. Cembrano, J. Quevedo, V. Puig, R. Perez, J. Figueras, J. M. Verdejo, I. Escaler, G. Ramon, G. Barnet, P. Rodriguez, and M. Casas. PLIO: a generic tool for realtime operational predictive optimal control of water networks. Water Science \& Technology, 64(2):448-459, 2011.

A. El Mouatasim, R. Ellaia, and A. Al-Hossain. A continuous approach to combinatorial optimization: application of water system pump operations. Optimization Letters, 6:177-198, 2012. ISSN 1862-4472.

E.G. Gilbert, I. Kolmanovsky, and K.T. Tan. Command governor for nonlinear systems under constraints. International Journal on Robust and Nonlinear Control, 5:487-504, 1995.

J.M. Grosso, C. Ocampo-Martinez, and V. Puig. Learningbased tuning of supervisory model predictive control for drinking water networks. Engineering Applications of Artificial Intelligence, 26(7):1741 - 1750, 2013.

P. Kapasouris, M. Athans, and G. Stein. Design of feedback control systems for unstable plants with saturating actuators. In Proc. of IFAC Symp. on Nolinear Control System Design, 1990.

J. Löfberg. Yalmip : A toolbox for modeling and optimization in MATLAB. In Proceedings of the CACSD Conference, Taipei, Taiwan, 2004. URL http://users.isy.liu.se/johanl/yalmip.

J.M. Maciejowski. Predictive control with constraints. Prentice Hall, Essex, England, 2002.

C. Ocampo-Martinez, V. Puig, G. Cembrano, R. Creus, and M. Minoves. Improving water management efficiency by using optimization-based control strategies: the Barcelona case study. Water Science \& Technology: Water supply, 9(5):565-575, 2009.

C. Ocampo-Martinez, V. Puig, G. Cembrano, and J. Quevedo. Application of predictive control strategies to the management of complex networks in the urban water cycle. Control Systems, IEEE, 33(1):15-41, 2013a.

C. Ocampo-Martinez, V. Puig, J.M. Grosso, and S. Montes de Oca. DMPC made easy, chapter Multi-layer Decentralized Model Predictive Control of Large-Scale Networked Systems. Springer, 2013b. To appear.

I. Pulido-Calvo and J.C. Gutiérrez-Estrada. Selection and operation of pumping stations of water distribution systems. Envirnomental Research Journal, 5:1-20, 2011.

I. Pulido-Calvo, J. Gutiérrez-Estrada, and D. Savic. Heuristic modelling of the water resources management in the Guadalquivir river basin, southern Spain. Water Resources Management, 26:185-209, 2012. ISSN 09204741.

J. Vieira, M.C. Cunha, L. Nunes, J.P. Monteiro, L. Ribeiro, T. Stigter, J. Nascimento, and H. Lucas. Optimization of the operation of large-scale multisource water-supply systems. Journal of Water Resources Planning and Management, 137(2):150-161, 2011. 